\title{
Biological and molecular characterizations of fluxapyroxad-resistant isolates of Botrytis cinerea
}

\author{
Kai Liu, Ziyue Wen, Zhonghua Ma and Wenyong Shao* (1)
}

\begin{abstract}
Gray mold caused by Botrytis cinerea leads to serious losses in various crops. Fluxapyroxad, a succinate dehydrogenase inhibitor (SDHI) fungicide, has been used to control gray mold for several years in China. In this study, we identified six fluxapyroxad-resistant (FluR) isolates from 96 isolates of B. cinerea collected in fields. Phenotypic assays showed that the FluR isolates exhibited defects in mycelial growth, conidiation, sclerotium formation, stress tolerance and virulence. Analysis of nucleotide sequences of succinate dehydrogenase subunit-coding genes revealed that various point mutations, including P225F, N230l, K283N and H272R in BcSdhB, G37S and P80H in BcSdhC, and V9A in BcSdhD, were associated with the resistance to fluxapyroxad in $B$. cinerea. Sensitivity assays showed that the FluR isolates also exhibited resistance to another SDHI boscalid, but there was no cross-resistance between fluxapyroxad and other fungicides with different modes of action. These results indicate that the resistance of $B$. cinerea to fluxapyroxad has occurred in fields, and further monitoring and management of the resistance to fluxapyroxad in B. cinerea should be carried out.
\end{abstract}

Keywords: Botrytis cinerea, Gray mold, Fluxapyroxad, Resistance

\section{Background}

Gray mold caused by Botrytis cinerea is an important plant disease that seriously threatens the yield and quality of economically important crops, including vegetables, fruits and ornamental plants (Williamson et al. 2007). To date, the common method for controlling gray mold worldwide is the application of chemical fungicides (Hahn 2014; Fan et al. 2017). Unfortunately, B. cinerea has been considered a plant pathogen with a high resistance-risk against fungicides due to its wide host range, fast growth rate, strong reproductive capacity and high genetic variability (Leroux et al. 2010). To date, B. cinerea populations collected from different crop fields have

*Correspondence: swy2012102114@163.com

State Key Laboratory of Rice Biology, Key Laboratory of Molecular Biology of Crop Pathogens and Insects, Institute of Biotechnology, Zhejiang

University, Hangzhou 310058, China exhibited resistance to various fungicides with different modes of action, including benzimidazole carbamates, dicarboximides, anilinopyrimidines, quinone outside inhibitors (QoIs), succinate dehydrogenase inhibitors (SDHIs) and phenylpyrroles (Leroux et al. 2002; Williamson et al. 2007; Fernández-Ortuño et al. 2013; Yin et al. 2018; Shao et al. 2021). The resistance has led to a decrease or a failure in control efficiency in the management of gray mold when using the aforementioned fungicides.

Fluxapyroxad is an SDHI fungicide produced by the BASF Corporation. It binds to the succinate dehydrogenase complex (Sdh, complex II) of the respiratory chain and disrupts cellular respiration (Lin et al. 2021). Fluxapyroxad has been reported to inhibit mycelial growth, germ tube development, spore germination and appressorium formation (Yang et al. 2019). Owing to its broad-spectrum and excellent antifungal activity, 
fluxapyroxad has been used to control various crop diseases caused by fungi (Li et al. 2020). The Sdh complex constitutes four subunits, SdhA, SdhB, SdhC and SdhD (Hägerhäll 1997), and amino acid mutations in SdhB, SdhC and SdhD lead to resistance to SDHIs in B. cinerea (Sierotzki and Scalliet 2013; Veloukas et al. 2013). Moreover, in B. cinerea, P225L/F/T, N230I or $\mathrm{H} 272 \mathrm{~L} / \mathrm{R} / \mathrm{Y}$ mutations in SdhB endows different resistance levels to boscalid (a commonly used SDHI), and the H132R mutation in SdhD is also responsible for the resistance to SDHIs (Leroux et al. 2010; Shao et al. 2021). Additionally, different amino acid mutations in SdhC were identified in field isolates of B. cinerea; these mutations are responsible for the decreased sensitivity of B. cinerea to SDHIs (Shao et al. 2020). Furthermore, compared with sensitive isolates, boscalid-resistant isolates containing single amino-acid mutations (H272L/R/Y, P225F or N230) in SdhB have decreased virulence on host plants (Veloukas et al. 2013). Yin et al. (2018) reported that boscalid-resistant isolates containing the H272R mutation in SdhB did not exhibit significant differences in mycelial growth, osmotic sensitivity and virulence compared with boscalid-sensitive isolates. To date, various SDHIs have been registered to control gray mold in fields, and therefore, investigation into the development of resistance of B. cinerea to SDHIs is essential for the management of gray mold.

In China, fluxapyroxad was registered to control fungal diseases including gray mold in 2014, and the mixture of fungicide products containing fluxapyroxad was subsequently used to spray twice to control gray mold during growth of greenhouse tomato plants. The application of mixed fungicide products containing fluxapyroxad for years has placed selective pressure on $B$. cinerea to generate resistance to this fungicide. To investigate the occurrence of fluxapyroxad resistance in B. cinerea, we collected $96 \mathrm{~B}$. cinerea isolates from tomato fields in four districts of Zhejiang Province in this study. The sensitivity of all these isolates to fluxapyroxad was analyzed via the discriminative method (Yin et al. 2018), and six FluR isolates were identified. Moreover, the fitness of the six FluR isolates was evaluated by determining the mycelial growth rate, conidiation capability and spore germination rate, sclerotium formation, stress tolerance and virulence. Additionally, we investigated the molecular characteristics of subunits within the succinate dehydrogenase (Sdh) complex in FluR isolates. The results lay the basis for management of resistance of $B$. cinerea to fluxapyroxad, and also for further understanding of the underlying resistance mechanism of plant pathogens to SDHI fungicides.

\section{Results}

Frequency and level of resistance to fluxapyroxad in $B$. cinerea isolates

To determine the sensitivity of $B$. cinerea to fluxapyroxad, we collected $96 \mathrm{~B}$. cinerea isolates from four districts in Zhejiang Province, China in 2020. Among them, six isolates exhibited resistance to fluxapyroxad. The resistance frequency of $B$. cinerea to fluxapyroxad was $6.25 \%$ (Table 1). These six FluR isolates (JS-16R, NB13R, NB-20R, XS-15R, XS-17R and YH-18R) and six randomly selected fluxapyroxad-sensitive (FluS) isolates (JS-5S, NB-10S, NB-19S, XS-12S, XS-23S and YH-27S) were used for further studies. The $\mathrm{EC}_{50}$ values of the six FluS isolates ranged from 0.2014 to $0.2505 \mu \mathrm{g} / \mathrm{mL}$; however, the $\mathrm{EC}_{50}$ values of the six FluR isolates ranged from 5.2472 to $38.5692 \mu \mathrm{g} / \mathrm{mL}$ (Table 2). Moreover, the six FluR isolates also exhibited resistance to boscalid, whereas the FluR isolates and FluS isolates showed similar sensitivity to tebuconazole, fluazinam and fludioxonil (Table 2), indicating that positive cross-resistance occurred between fluxapyroxad and boscalid but not between fluxapyroxad and tebuconazole, fluazinam or fludioxonil.

\section{FluR isolates exhibit defects in mycelial growth, conidiation, spore germination and sclerotium formation} To detect if there are differences in growth and reproductive characteristics between FluR and FluS isolates of $B$. cinerea, we carried out investigations on mycelial growth rate, conidiation, spore germination and sclerotium formation. The results of mycelial growth assay showed that there was no difference in mycelial growth rate between FluR and FluS isolates on PDA; however, compared with that of FluS isolates, the mycelial growth rate of FluR isolates was obviously decreased on MM (Fig. 1a-d). For conidiation assay, all the tested isolates generated vast amounts of conidia on both PDA and MM; however, the conidiation capability of FluR isolates was reduced compared with that of FluS isolates (Fig. 1e, f). For spore germination assay, the spore germination rate of FluR

Table 1 Frequency and distribution of FluR isolates from different districts in Zhejiang Province, China

\begin{tabular}{llll}
\hline Location & $\begin{array}{l}\text { The number of } \\
\text { isolates tested }\end{array}$ & $\begin{array}{l}\text { The number of } \\
\text { resistant isolates }\end{array}$ & $\begin{array}{l}\text { Frequency of } \\
\text { resistant isolates } \\
\text { (\%) }\end{array}$ \\
\hline Jiashan & 19 & 1 & 5.26 \\
Ningbo & 26 & 2 & 7.69 \\
Xiasha & 23 & 2 & 8.69 \\
Yuhang & 28 & 1 & 3.57 \\
Total & 96 & 6 & 6.25 \\
\hline
\end{tabular}


Table 2 Sensitivity analysis of FluR and FluS isolates to fluxapyroxad, boscalid, fluazinam, fludioxonil and tebuconazole

\begin{tabular}{|c|c|c|c|c|c|c|c|c|c|c|c|c|}
\hline \multirow[t]{2}{*}{ Isolates } & \multirow[t]{2}{*}{ Sensitivity } & \multirow[t]{2}{*}{ Origin } & \multicolumn{2}{|l|}{ Fluxapyroxad } & \multicolumn{2}{|l|}{ Boscalid } & \multicolumn{2}{|l|}{ Fluazinam } & \multicolumn{2}{|l|}{ Fludioxonil } & \multicolumn{2}{|l|}{ Tebuconazole } \\
\hline & & & $\mathrm{EC}_{50}(\mu \mathrm{g} / \mathrm{mL})$ & $\mathrm{RF}$ & $\mathrm{EC}_{50}(\mu \mathrm{g} / \mathrm{mL})$ & $\mathrm{RF}$ & $\mathrm{EC}_{50}(\mu \mathrm{g} / \mathrm{mL})$ & $\mathrm{RF}$ & $\mathrm{EC}_{50}(\mu \mathrm{g} / \mathrm{mL})$ & $\mathrm{RF}$ & $\mathrm{EC}_{50}(\mu \mathrm{g} / \mathrm{mL})$ & RF \\
\hline JS-5S & S & Field & $0.2014 C$ & - & $0.7291 C$ & - & $0.0164 \mathrm{~A}$ & - & $0.0071 \mathrm{~A}$ & - & $0.2245 \mathrm{~A}$ & - \\
\hline NB-10S & S & Field & $0.2204 C$ & - & $0.9238 \mathrm{C}$ & - & $0.0183 \mathrm{~A}$ & - & $0.0078 \mathrm{~A}$ & - & $0.2236 \mathrm{~A}$ & - \\
\hline NB-19S & S & Field & $0.2505 C$ & - & $0.7234 \mathrm{C}$ & - & $0.0156 \mathrm{~A}$ & - & $0.0068 \mathrm{~A}$ & - & $0.2644 \mathrm{~A}$ & - \\
\hline$X S-12 S$ & S & Field & $0.2239 C$ & - & $0.6919 C$ & - & $0.0193 \mathrm{~A}$ & - & $0.0066 \mathrm{~A}$ & - & $0.2273 \mathrm{~A}$ & - \\
\hline XS-23S & S & Field & $0.2438 C$ & - & $0.7620 \mathrm{C}$ & - & $0.0188 \mathrm{~A}$ & - & $0.0081 \mathrm{~A}$ & - & $0.2266 \mathrm{~A}$ & - \\
\hline $\mathrm{YH}-27 \mathrm{~S}$ & S & Field & $0.2127 C$ & - & $0.7486 C$ & - & $0.0121 \mathrm{~A}$ & - & $0.0069 \mathrm{~A}$ & - & $0.2587 \mathrm{~A}$ & - \\
\hline$J S-16 R$ & $\mathrm{R}$ & Field & $38.5692 \mathrm{~A}$ & 171.08 & $45.3007 \mathrm{~A}$ & 59.36 & $0.0112 \mathrm{~A}$ & 0.67 & $0.0079 \mathrm{~A}$ & 1.09 & $0.2623 \mathrm{~A}$ & 1.10 \\
\hline NB-13R & $R$ & Field & $5.5389 \mathrm{~B}$ & 24.57 & 9.5309 B & 12.49 & $0.0178 \mathrm{~A}$ & 1.06 & $0.0073 \mathrm{~A}$ & 1.01 & $0.2526 \mathrm{~A}$ & 1.06 \\
\hline NB-20R & $R$ & Field & 5.2472 B & 23.27 & 9.3567 B & 12.26 & $0.0176 \mathrm{~A}$ & 1.05 & $0.0076 \mathrm{~A}$ & 1.05 & $0.2268 \mathrm{~A}$ & 0.95 \\
\hline XS-15R & R & Field & 5.3613 B & 23.78 & 9.5774 B & 12.55 & $0.0137 \mathrm{~A}$ & 0.82 & $0.0067 \mathrm{~A}$ & 0.93 & $0.2552 \mathrm{~A}$ & 1.07 \\
\hline XS-17R & R & Field & $5.6009 \mathrm{~B}$ & 24.84 & 9.2296 B & 12.09 & $0.0172 \mathrm{~A}$ & 1.03 & $0.0078 \mathrm{~A}$ & 1.08 & $0.2537 \mathrm{~A}$ & 1.07 \\
\hline YH-18R & R & Field & $5.6381 \mathrm{~B}$ & 25.01 & 9.5973 B & 12.58 & $0.0163 \mathrm{~A}$ & 0.97 & $0.0082 \mathrm{~A}$ & 1.14 & $0.2276 \mathrm{~A}$ & 0.96 \\
\hline
\end{tabular}

$\mathrm{RF}$, resistance factor, the ratio of $\mathrm{EC}_{50}$ of a FluR isolate relative to the mean value of $\mathrm{EC}_{50}$ of FluS isolates

$S$ indicates an isolate is sensitive to fluxapyroxad; $R$ indicates an isolate is resistant to fluxapyroxad

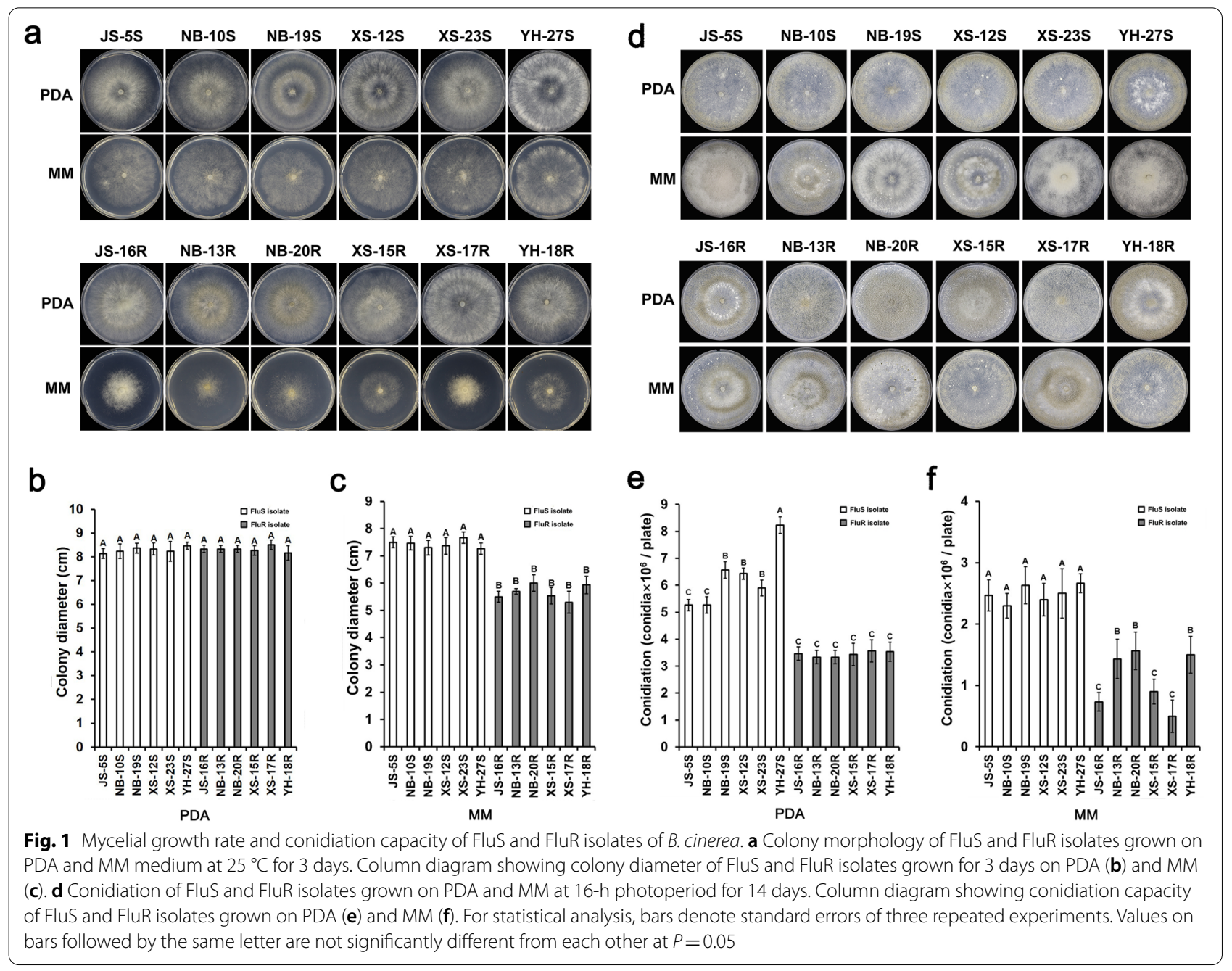


isolates was significantly lower than that of FluS isolates on WA; however, the spore germination rate of FluR isolates was obviously higher than that of FluS isolates on WA supplemented with $5 \mu \mathrm{g} / \mathrm{mL}$ fluxapyroxad (Fig. 2a, b). In sclerotium formation assay, we found that the number of sclerotia produced by FluR isolates was lower than that produced by FluS isolates (Fig. 2c, d). These results indicate that compared with FluS isolates, FluR isolates exhibit defects in mycelial growth, conidiation, spore germination and sclerotium formation, and as expected, FluR isolates also exhibit resistance to fluxapyroxad during spore germination.

FluR isolates have increased sensitivity to multiple stresses and decreased virulence on host plants

To determine if the stress tolerance of FluR isolates is changed compared with FluS isolates, the stress tolerance assay was performed on PDA medium supplemented with Congo red, $\mathrm{KCl}, \mathrm{NaCl}$ or $\mathrm{H}_{2} \mathrm{O}_{2}$, and the results showed that the mycelial growth inhibition rate of FluR isolates was significantly higher than that of FluS isolates under all these stress conditions (Fig. 3). For the virulence assay, each isolate caused obvious lesions on apple and tomato; however, FluR isolates caused obviously smaller disease spots than FluS isolates (Fig. 4). These results indicate that FluR isolates exhibit increased sensitivity to various stresses and decreased virulence on host plants.

\section{FluR isolates have multiple types of mutation in subunits of succinate dehydrogenase complex}

To investigate the molecular mechanism underlying the resistance of $B$. cinerea to fluxapyroxad, we compared nucleotide sequences coding for the subunits of succinate dehydrogenase, the target of SDHI fungicides, between FluR and FluS isolates, and seven types of mutations were identified in FluR isolates. The different types of mutations in FluR isolates were as follows: $\mathrm{BcSdh}^{\mathrm{P} 225 \mathrm{~F}}$ in JS-16R; $\mathrm{BcSdhB}^{\mathrm{N} 230 \mathrm{I}}$ in NB-13R; $\mathrm{BcSdhB}^{\mathrm{N} 230 \mathrm{I}}$ and $\mathrm{BcSdhD}^{\mathrm{V} 9 \mathrm{~A}}$ in NB-20R; $\mathrm{BcSdh}^{\mathrm{N} 230 \mathrm{I}}, \mathrm{BcSdhB}^{\mathrm{K} 283 \mathrm{~N}}$ and $\mathrm{BcSdhD}^{\mathrm{V} 9 \mathrm{~A}}$ in $\mathrm{XS}-15 \mathrm{R}$; $\mathrm{BcSdhC}^{\mathrm{G} 37 \mathrm{~S}}$ in $\mathrm{XS}-17 \mathrm{R}$; and $\mathrm{BCSdhB}^{\mathrm{H} 272 \mathrm{R}}$ and $\mathrm{BCSdhC} \mathrm{C}^{\mathrm{P} 80 \mathrm{H}}$ in $\mathrm{YH}-18 \mathrm{R}$ (Table 3).

\section{Discussion}

Fluxapyroxad, an important SDHI fungicide, has been used to control gray mold for several years. In general, continuous application of a fungicide on a certain plant pathogenic fungus will promote the generation of resistant fungal isolates against this fungicide, and as a result, the resistant isolates may gradually develop into dominant populations that can lead to serious crop losses (Yin et al. 2018). To investigate whether FluR isolates of $B$. cinerea have emerged in the tomato fields where fluxapyroxad were used to control gray mold, our study focused on monitoring and characterizing FluR isolates of $B$. cinerea. Our data indicated that a total of six FluR isolates were identified among 96 isolates collected from fields in four districts in Zhejiang Province, China. As is well known, fitness is an extremely essential parameter for evaluating the potential risk of fungicide-resistant populations (Wang et al. 2021). In current study, the FluR isolates exhibited defects in mycelial growth, conidiation, spore germination, sclerotium formation, stress tolerance and virulence compared with FluS isolates, indicating that the fitness of FluR isolates is weaker than that of FluS isolates, and therefore the potential risk for FluR isolates to develop into dominant populations is low currently. However, the $6.25 \%$ distribution frequency for FluR isolates is still relatively high; furthermore, FluR isolates were found in all the sampled regions, indicating that fluxapyroxad-resistant $B$. cinerea strains had been widely distributed in tomato fields. Therefore, it is necessary to seek some resistance management measures to help avoid or delay the development of FluR B. cinerea populations, including enhancing resistance monitoring, mixing or alternating application of fluxapyroxad and other fungicides with different modes of action (MoA). In previous studies, many $B$. cinerea isolates were found to exhibit multiple drug resistance (Shao et al. 2021), and it looks like alternation of fungicides with different MoA could speed up selection for multi-resistance in $B$. cinerea, thus, for management of fluxapyroxad resistance, the frequency and dosage of fungicides with different MoA should be scientifically evaluated and applied.

Previous studies have shown that the amino acid mutations $\mathrm{SdhB}^{\mathrm{P} 225 \mathrm{~L} / \mathrm{F} / \mathrm{T} \text {, N230I or } \mathrm{H} 272 \mathrm{~L} / \mathrm{R} / \mathrm{Y}}$ and $\mathrm{SdhD}^{\mathrm{H} 132 \mathrm{R}}$ are associated with the resistance of $B$. cinerea to SDHIs (Sierotzki and Scalliet 2013; Veloukas et al. 2013; Shao et al. 2021), and amino acid substitutions in SdhC are also responsible for the decreased sensitivity of $B$. cinerea to SDHIs (Shao et al. 2020). Moreover, it was reported that various mutations are involved in different resistance level of B. cinerea to SDHIs, including $\mathrm{SdhB}^{\mathrm{N} 230 \mathrm{I}}$ and $\mathrm{Sdh}^{\mathrm{H} 272 \mathrm{R} / \mathrm{Y}}$, which were found in moderately boscalid-resistant isolates, and $\mathrm{SdhB}^{\mathrm{H} 272 \mathrm{~L}}$ and $\mathrm{SdhB}^{\mathrm{P} 225 \mathrm{~F} / \mathrm{L} \text {, }}$

(See figure on next page.)

Fig. 2 Conidia germination rate and sclerotium production of FluS and FluR isolates of B. cinerea. The conidia germination rate of FluS and FluR isolates incubated at $25^{\circ} \mathrm{C}$ for $10 \mathrm{~h}$ on WA (a) and WA supplemented with $5 \mathrm{\mu g} / \mathrm{mL}$ fluxapyroxad (+ Flu) (b). c Observation of sclerotium production of FluS and FluR isolates grown on PDA for 3 days at $25^{\circ} \mathrm{C}$ and then incubated for 4 weeks at $10^{\circ} \mathrm{C}$. $\mathbf{d}$ Column diagram showing the amount of sclerotia of FluS and FluR isolates after incubation on PDA for 3 days at $25^{\circ} \mathrm{C}$ and then for 4 weeks at $10^{\circ} \mathrm{C}$. For statistical analysis, bars denote standard errors of three repeated experiments. Values on bars followed by the same letter are not significantly different from each other at $P=0.05$ 

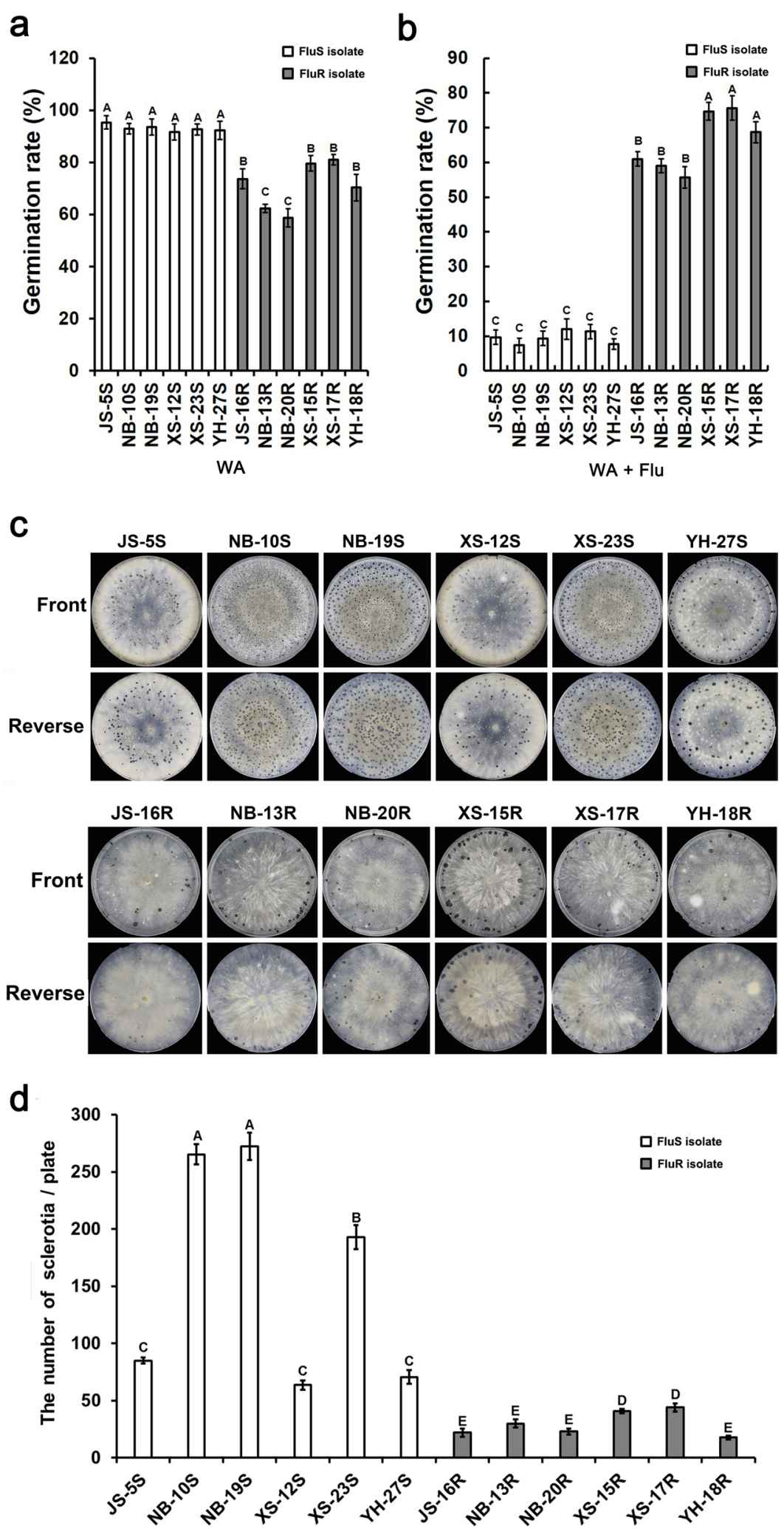

Fig. 2 (See legend on previous page.) 
which were identified in highly boscalid-resistant isolates (Veloukas et al. 2013). In this study, seven different mutations, including $\mathrm{BcSdhB}^{\mathrm{P} 225 \mathrm{~F}}, \mathrm{BcSdh}^{\mathrm{H} 272 \mathrm{R}}$, $\mathrm{BcSdhB}^{\mathrm{K} 283 \mathrm{~N}}, \mathrm{BcSdh}^{\mathrm{N} 230 \mathrm{I}}, \mathrm{BcSdh}^{\mathrm{G} 37 \mathrm{~S}}, \mathrm{BcSdhC}^{\mathrm{P} 80 \mathrm{H}}$ and $\mathrm{BcSdhD}^{\mathrm{V} 9 \mathrm{~A}}$ were identified in FluR isolates. Among them, $\mathrm{BcSdh}^{\mathrm{K} 283 \mathrm{~N}}, \mathrm{BcSdhC}^{\mathrm{P} 80 \mathrm{H}}, \mathrm{BcSdhC}^{\mathrm{G} 37 \mathrm{~S}}$ and $\mathrm{BcSdhD}^{\mathrm{V} 9 \mathrm{~A}}$ are new types of mutation that were identified for the first time in SDHI-resistant $B$. cinerea isolates. Before the application of fluxapyroxad, another SDHI fungicide boscalid was ever used to control gray mold in these sampled tomato fields where FluR isolates were obtained. Therefore, we infer that the mutations $\mathrm{BcSdhB}^{\mathrm{P} 225 \mathrm{~F}}$, $\mathrm{BcSdhB}^{\mathrm{H} 272 \mathrm{R}}$ and $\mathrm{BcSdh}^{\mathrm{N} 230 \mathrm{I}}$ might have existed in $B$. cinerea before the application of fluxapyroxad and are induced by boscalid, whereas the selective pressure exerted by fluxapyroxad leads to the generation of new types of mutation including $\mathrm{BcSdh} \mathrm{B}^{\mathrm{K} 283 \mathrm{~N}}, \mathrm{BcSdhC}^{\mathrm{P} 80 \mathrm{H}}$, $\mathrm{BcSdhC}^{\mathrm{G} 37 \mathrm{~S}}$ and $\mathrm{BcSdhD}^{\mathrm{V9A}}$. In sensitivity assays of $B$. cinerea isolates to fluxapyroxad and boscalid, we found that the resistance factor (RF) of JS-16R containing the $\mathrm{BcSdhB}^{\mathrm{P} 225 \mathrm{~F}}$ mutation was higher than that of other FluR isolates, and there was no difference in RF among NB13R, NB-20R, XS-15R, XS-17R and YH-18R (Tables 1, 2). Lalève et al. (2014) also reported that the $\mathrm{BcSdh}^{\mathrm{P} 225 \mathrm{~F}}$ mutation in $B$. cinerea confers a high-level resistance to boscalid and fluopyram (2014). These results indicate that the $\mathrm{BcSdhB}^{\mathrm{P} 225 \mathrm{~F}}$ mutation in B. cinerea is responsible for a high-level resistance to fluxapyroxad, boscalid and fluopyram. It was found that mutations in BcSdhB could decrease Sdh inhibition, leading to the resistance to SDHIs, and different BcSdhB modifications exhibit differences in interfering with SDHI binding (Lalève et al. 2014). Accordingly, we consider that the mutation $\mathrm{BcSdh}^{\mathrm{P} 225 \mathrm{~F}}$ confers a higher resistance to fluxapyroxad in $B$. cinerea than other types of mutation due to that it causes a more serious defect in the binding activity of fluxapyroxad to Sdh. Moreover, mutations in BcSdhB or BcSdhC were demonstrated to affect Sdh activity, respiration and carbon source utilization (Lalève et al. 2014; Shao et al. 2020); thus, we infer that those mutations in FluR isolates may also affect Sdh activity, respiration and carbon source utilization, leading to defects in mycelial growth, conidiation, spore germination, sclerotium formation, stress tolerance and virulence. Additionally, inconsistent with this study, previous studies found much difference in fitness of the SDHIs-resistant isolates (Yin et al. 2018; Petrasch et al. 2019; Shao et al. 2021), and the variation in fitness cost among SDHIs-resistant isolates might be due to the differences in some specific mutations.

In this study, several FluR isolates were found to include different types of mutation: $\mathrm{BcSdhB}^{\mathrm{N} 230 \mathrm{I}}$ and $\mathrm{BCSdhD}^{\mathrm{V} 9 \mathrm{~A}}$ were found in $\mathrm{NB}-20 \mathrm{R} ; \mathrm{BcSdhB}^{\mathrm{N} 230 \mathrm{I}}$, $\mathrm{BcSdhB}^{\mathrm{K} 283 \mathrm{~N}}$ and $\mathrm{BcSdhD}{ }^{\mathrm{V} 9 \mathrm{~A}}$ were identified in XS-15R; $\mathrm{BcSdhB}^{\mathrm{H} 272 \mathrm{R}}$ together with $\mathrm{BcSdh} \mathrm{C}^{\mathrm{P} 80 \mathrm{H}}$ were identified in $\mathrm{YH}-18 \mathrm{R}$. However, there is no difference in resistance level to fluxapyroxad among these three FluR isolates. From the perspective of resistance evolution, we conclude that one or two mutations in these FluR isolates are single nucleotide polymorphisms that are not associated with the resistance of $B$. cinerea to SDHIs. This is the first report of multiple different mutations existing in a single SDHI-resistant $B$. cinerea isolate. The biological mechanism behind a single resistant isolate generating multiple different mutations is worth exploring.

\section{Conclusions}

We collected $96 \mathrm{~B}$. cinerea field isolates, of which six exhibited resistance to fluxapyroxad. Moreover, all FluR isolates exhibited defect in fitness. There was no crossresistance between fluxapyroxad and other fungicides with different modes of action, including tebuconazole, fluazinam or fludioxonil. Moreover, new types of mutations, $\mathrm{BcSdh}^{\mathrm{K} 283 \mathrm{~N}}, \mathrm{BcSdh}^{\mathrm{P} 80 \mathrm{H}}, \mathrm{BcSdhC}^{\mathrm{G} 37 \mathrm{~S}}$ and $\mathrm{BcSdhD}^{\mathrm{V9A}}$, were identified in FluR isolates. These results provide directions for the management of fluxapyroxad resistance in B. cinerea and further improve our understanding of the underlying resistance mechanism of plant pathogens to SDHIs.

\section{Methods}

\section{Culture media and fungicides}

Culture media used in this study include potato dextrose agar (PDA, $200 \mathrm{~g}$ of potato, $20 \mathrm{~g}$ of dextrose and $18 \mathrm{~g}$ agar per liter of distilled water), potato dextrose broth (PDB, $200 \mathrm{~g}$ of potato and $20 \mathrm{~g}$ of dextrose per liter of distilled water), alkylester agar medium (AEA, $5 \mathrm{~g}$ of yeast extract, $6 \mathrm{~g}$ of $\mathrm{NaNO}_{3}, 1.5 \mathrm{~g}$ of $\mathrm{KH}_{2} \mathrm{PO}_{4}, 0.5 \mathrm{~g}$ of $\mathrm{KCl}, 0.25 \mathrm{~g}$ $\mathrm{MgSO}_{4}, 20 \mathrm{~mL}$ of glycerol and $20 \mathrm{~g}$ of agar per liter of distilled water).

Technical grades of fluxapyroxad (96.5\%), boscalid (98\%) fluazinam (98\%), fludioxonil (95\%) and tebuconazole (95\%) were provided by Badische Anilin Soda Fabrik Ga (Germany), Jingbo Agrochemical Technology Co. LTD, Zhejiang Hetian Chemical Co., LTD, Qingdao Runnong Chemical Co., LTD and Jiangsu Changfeng

(See figure on next page.)

Fig. 3 Sensitivity of FluS and FluR isolates of B. cinerea to various stresses. a Colony morphology of FluS and FluR isolates grown on PDA modified with or without stress agents for 3 days. The relative growth rate of FluS and FluR isolates grown for 3 days on PDA supplemented with $30 \mathrm{mg} / \mathrm{mL}$ Congo red (b), 1.2 M KCl (c), $1.0 \mathrm{M} \mathrm{NaCl}(\mathbf{d})$ or $24 \mathrm{mM} \mathrm{H}_{2} \mathrm{O}_{2}$ (e). For statistical analysis, bars denote standard errors of three repeated experiments. Values on bars followed by the same letter are not significantly different from each other at $P=0.05$ 


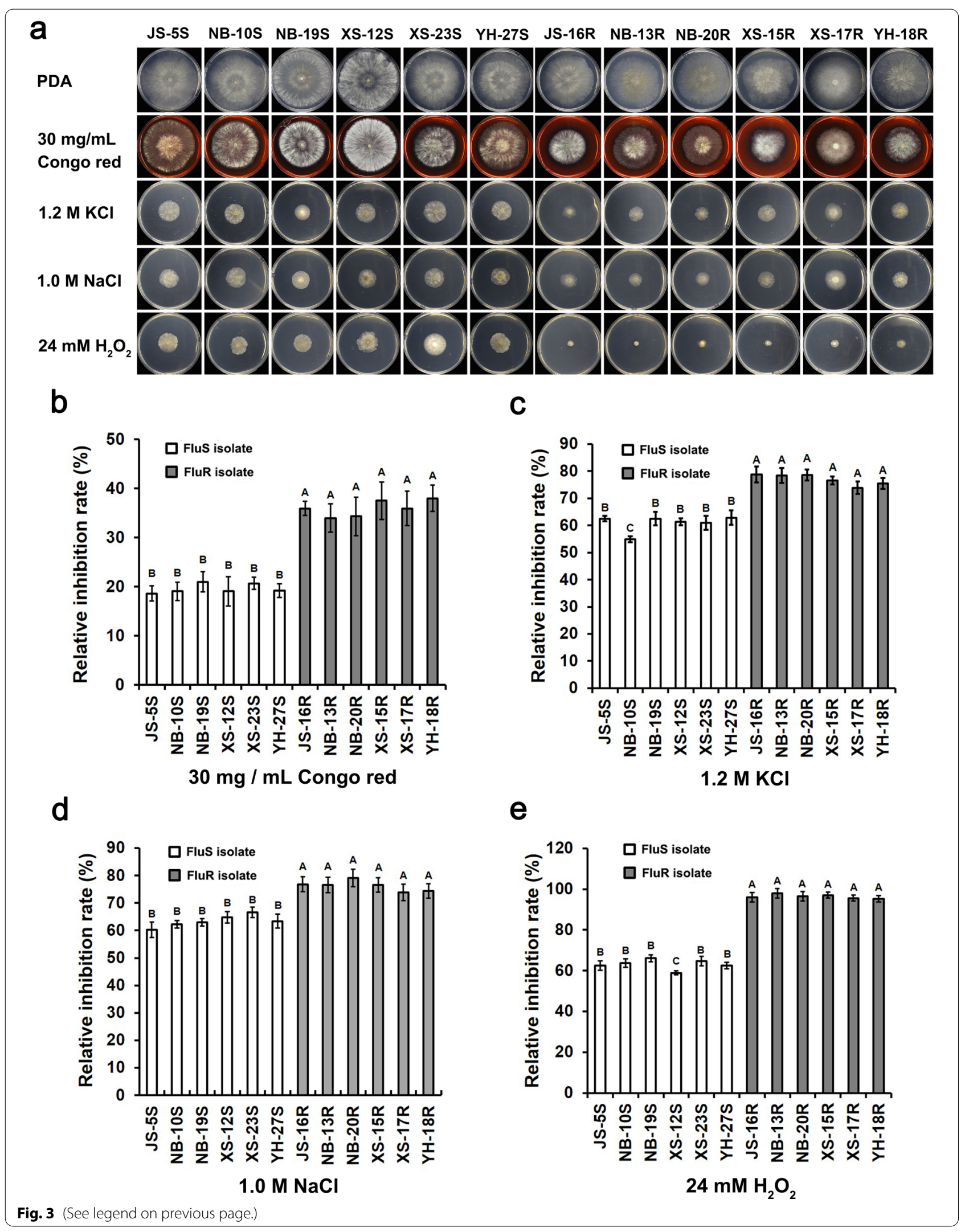




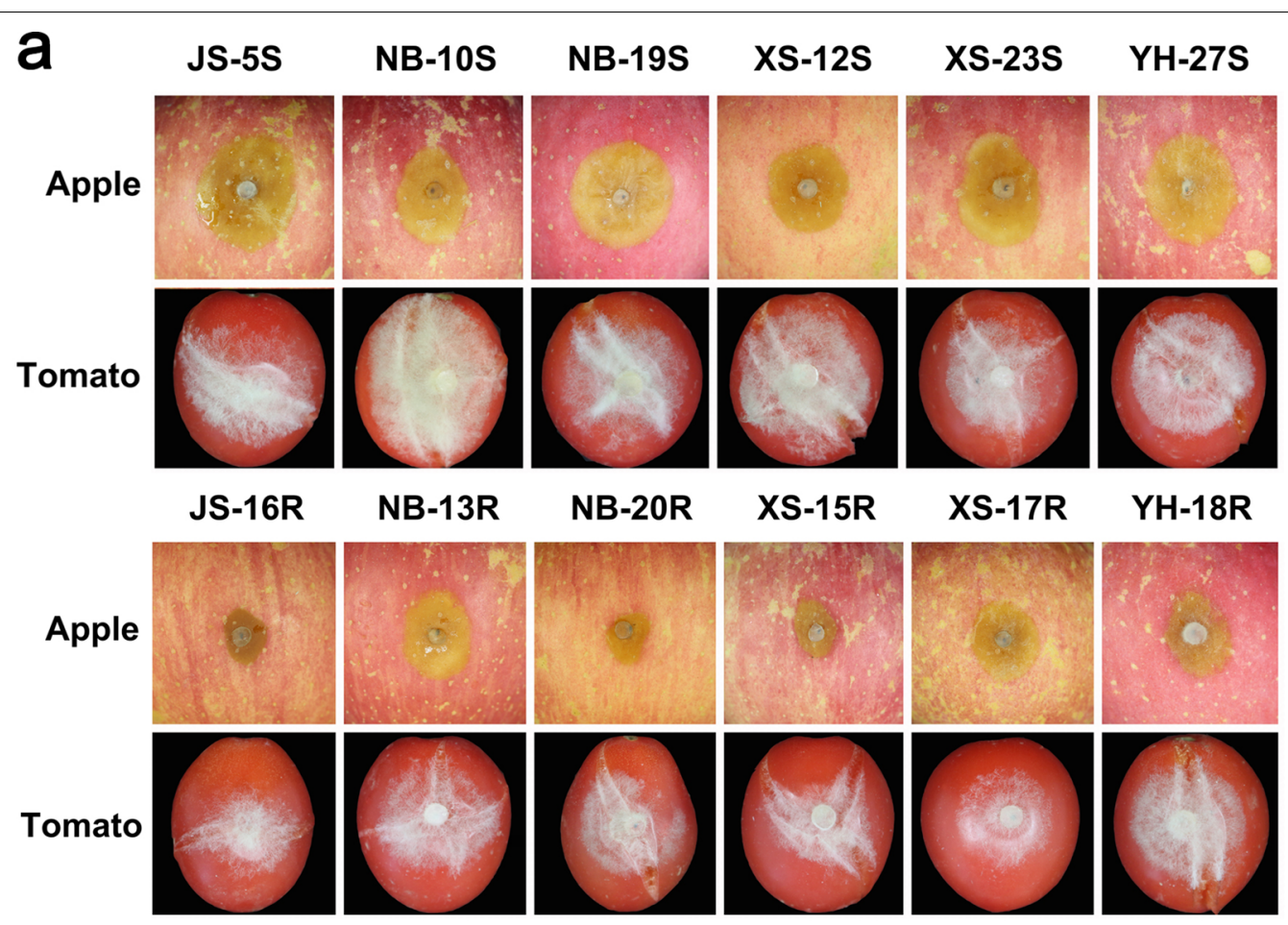

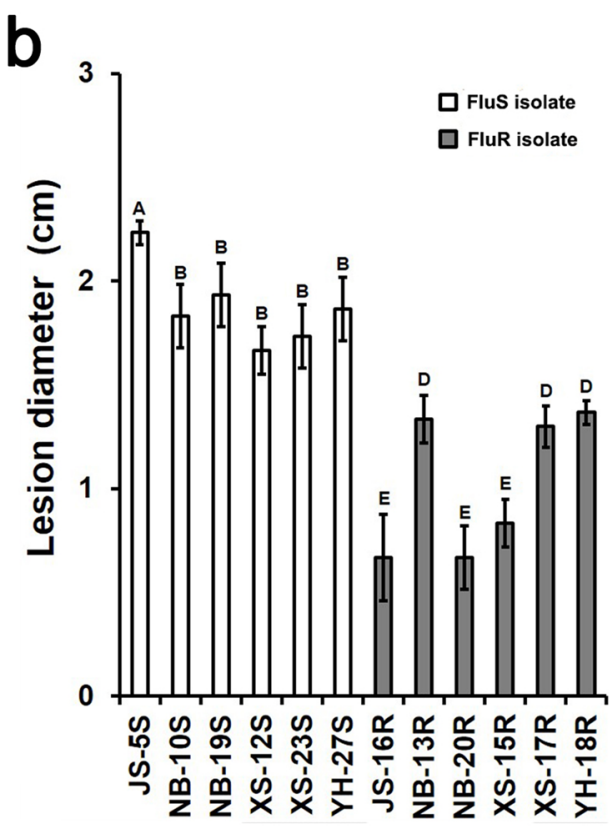

Inoculation on apple

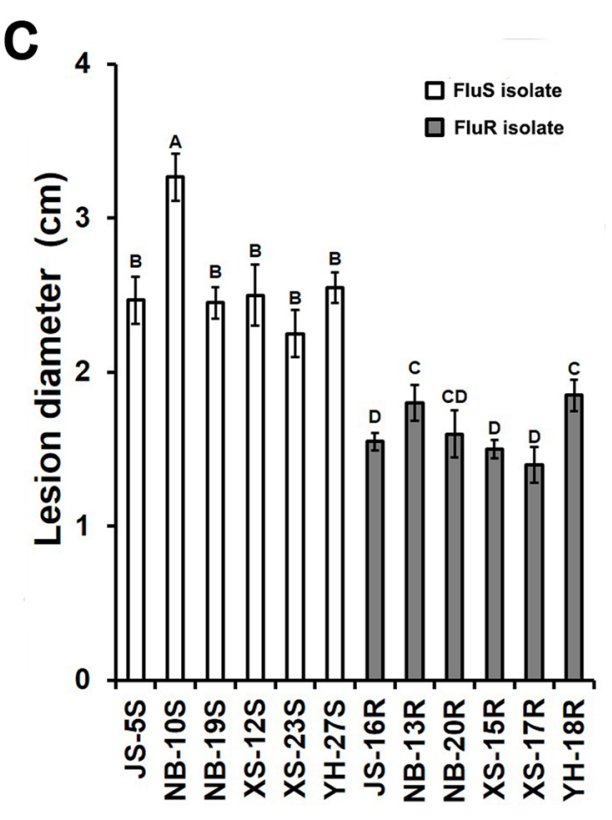

Inoculation on tomato

Fig. 4 Virulence assay of FluS and FluR isolates of B. cinerea on apple and tomato fruits. a Symptoms on wounded apple and tomato fruits after inoculated with B. cinerea isolates for $72 \mathrm{~h}$. Lesion diameter measured at $72 \mathrm{~h}$ post-inoculation (hpi) on apple fruits (b) and tomato fruits (c). For statistical analysis, bars denote standard errors of three repeated experiments. Values on bars followed by the same letter are not significantly different from each other at $P=0.05$ 
Table 3 Mutations in BcSdhB, BcSdhC and BcSdhD of FluR isolates

\begin{tabular}{llllll}
\hline Isolates & Origin & Sensitivity & SDHB sequence & SDHC sequence & SDHD sequence \\
\hline JS-5S, NB-10S, XS-12S & Field & $S$ & WT & WT & WT \\
JS-16R & Field & $R$ & CCC (P) 225 TTC (F) & & \\
NB-13R & Field & $R$ & AAC (N) 230 ATC (I) & & GTC (V) 9 GCC \\
NB-20R & Field & $R$ & AAC (N) 230 ATC (I) & & (A) \\
XS-15R & Field & $R$ & AAC (N) 230 ATC (I), AAG (K) & & (A) GCC \\
XS-17R & Field & R & 283 AAT(N) & GGC (G) 37 AGC (S) & CCT (P) 80 CAT (H) \\
YH-18R & Field & $R$ & CAC (H) 272 CGC (R) &
\end{tabular}

S indicates an isolate is sensitive to fluxapyroxad; $\mathrm{R}$ indicates an isolate is resistant to fluxapyroxad

Agrochemical Co. LTD. respectively. All fungicides were dissolved in methanol to obtain a stock solution containing $10 \mathrm{mg}$ active ingredient per $\mathrm{mL}$.

\section{Collection of $B$. cinerea isolates}

A total of 96 B. cinerea isolates were isolated from tomato plants at two locations in Jiashan (19), three locations in Ningbo (26), two locations in Xiasha (23) and three locations in Yuhang (28) in Zhejiang Province, China in 2020, with a distance of at least $0.5 \mathrm{~km}$ between different sampling sites within a location. In these sampling locations, Orkestra, a fluxapyroxad-containing fungicide, was used to control gray mold. To obtain B. cinerea isolates, small tissue fragments cut from lesion margins were disinfected in $1 \% \mathrm{NaClO}$ for $2 \mathrm{~min}$, washed three times with sterile water, and then put onto PDA medium supplemented with streptomycin sulfate at a final concentration of $100 \mu \mathrm{g} / \mathrm{mL}$. After incubation at $25^{\circ} \mathrm{C}$ for 3 days, mycelial plugs cut from the margins of colony were transferred onto fresh PDA medium and incubated at $25{ }^{\circ} \mathrm{C}$ under a $16 \mathrm{~h}$-photoperiod for 12 days to obtain conidia. All isolates were purified by single-spore isolation and then maintained on PDA slant at $4{ }^{\circ} \mathrm{C}$. In total, 96 single-spore isolates of $B$. cinerea were obtained from field-grown tomato plants.

\section{Assessment of sensitivity of $B$. cinerea isolates to fluxapyroxad and other fungicides}

To determine the sensitivity of $B$. cinerea isolates to fluxapyroxad, a mycelial plug cut from the edge of a 3-day-old colony was transferred onto AEA medium modified with fluxapyroxad at a discriminatory concentration of $5 \mu \mathrm{g} / \mathrm{mL}$, with three replicates for each isolate. After incubated for 3 days at $25^{\circ} \mathrm{C}$, the isolates that were able to grow were identified as potential FluR isolates, while those unable to grow were designated as FluS isolates. The resistance frequency was calculated via the following formula: Resistance frequency $(\%)=[($ Number of FluR isolates / Total number of isolates) $\times 100]$. To further measure sensitivity of FluR isolates to fluxapyroxad, the $\mathrm{EC}_{50}$ (effective concentration for $50 \%$ inhibition of mycelial growth) of six FluR isolates and six randomly selected FluS isolates were calculated. For each isolate, a $5 \mathrm{~mm}$ mycelial plug was cut from the edge of a 3 day-old-colony and put onto AEA medium modified with pydiflumetofen at a final concentration of $0.03125,0.0625,0.15625,1.25$, $2.5,5,10,20$ or $40 \mu \mathrm{g} / \mathrm{mL}$. After 3 days of incubation at $25{ }^{\circ} \mathrm{C}$ in the dark, the mycelial growth inhibition rate was measured and the $\mathrm{EC}_{50}$ value of each isolate was analyzed with DPS software as previously reported (Zhou et al. 1994; Chen et al. 2021; Zhang et al. 2021). $\mathrm{EC}_{50}$ of these isolates to other fungicide (boscalid, fluazinam, fludioxonil and tebuconazole) were also measured via the same method. The experiment was repeated independently for two times, with three replicates for each treatment.

\section{Measurement of mycelial growth rate, conidiation capacity, spore germination rate and sclerotia production of $B$. cinerea isolates}

For mycelial growth assay, a $5 \mathrm{~mm}$ mycelial plug was cut from the edge of an actively growing colony and put onto PDA or MM medium plates, with three replicates for each isolate on each culture medium. After 3 days of incubation at $25{ }^{\circ} \mathrm{C}$ in the dark, the diameter of colony was measured, and the original mycelial plug diameter $(5 \mathrm{~mm})$ was subtracted from measured data. The experiment was repeated independently for two times.

For conidiation assay, a mycelial plug was cut from the edge of a 3-day-old colony of each isolate and placed onto PDA or MM medium plates, with three replicates for each isolate on each culture medium. After incubated at $25^{\circ} \mathrm{C}$ for 12 days under a 16 -h photoperiod, conidia were

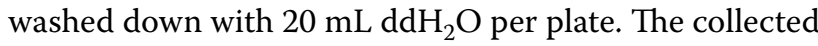
conidia suspension was filtrated with lens-wiping paper and centrifuged at $1,300 \times g$ for $5 \mathrm{~min}$, after which the

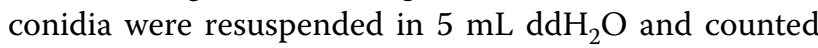
on a hemacytometer. The experiment was repeated independently for two times. 
For spore germination assay, spore suspension of each isolate was prepared as described above, and adjusted to a concentration of $10^{5} / \mathrm{mL}$. A volume of $200 \mu \mathrm{L}$ spore suspension was added and smeared evenly onto WA medium supplemented with or without $5 \mu \mathrm{g} / \mathrm{mL}$ of fluxapyroxad, with three replicates for each isolate. After incubated at $25^{\circ} \mathrm{C}$ for $10 \mathrm{~h}$ in darkness, the spore germination rate of each isolate was calculated by the following formula: Spore germination rate $(\%)=[$ (Number of germinated spores/Total number of spores $) \times 100]$. The experiment was repeated independently for two times.

To measure production of sclerotia, a mycelial plug cut from the edge of a 3-day-old colony of each isolate was transferred onto PDA medium, incubated at $25{ }^{\circ} \mathrm{C}$ for 3 days in darkness and then incubated for two weeks at $10{ }^{\circ} \mathrm{C}$. Finally, the amount of sclerotia produced by each isolate was measured. Three replicates for each treatment. The experiment was repeated two times.

\section{Determination of stress sensitivity and virulence of $B$. cinerea isolates}

To analyze stress sensitivity, a $5 \mathrm{~mm}$ mycelial plug cut from the edge of an actively growing colony and transferred onto PDA medium modified with $30 \mathrm{mg} / \mathrm{mL}$ of Congo red, $1.2 \mathrm{M} \mathrm{KCl}, 1.0 \mathrm{M} \mathrm{NaCl}$ or $24 \mathrm{mM} \mathrm{H}_{2} \mathrm{O}_{2}$, with three replicates for each treatment. PDA medium without stress agents was served as the control treatment. The diameter of colony was measured after incubated at $25^{\circ} \mathrm{C}$ for 3 days in darkness. The percentage of inhibition of mycelial radial growth (PIMG) was calculated using the following formula: PIMG $(\%)=[(\mathrm{C}-\mathrm{N}) /(\mathrm{C}-5)] \times 100$, in which $\mathrm{C}$ is the colony diameter of the untreated control and $\mathrm{N}$ is that of a treatment. The experiment was repeated independently for three times.

To measure virulence, all apple and tomato fruits were surface-sterilized with $75 \%$ alcohol, wounded with a sterilized needle and then inoculated with mycelial plugs cut from the edge of 3-day-old colony of each isolate, with five replicates for each isolate. The inoculated fruits were incubated at $25{ }^{\circ} \mathrm{C}$ and $90 \%$ relative humidity under a $16-\mathrm{h}$ photoperiod. The lesion diameter was measured at $72 \mathrm{hpi}$. The experiment was repeated independently for two times.

\section{Cloning and sequencing of target genes}

Genomic DNA of FluR and FluS isolates was extracted using the method described previously (Yin et al. 2011). The Sdh complex in B. cinerea contains four subunits including BcSdhA (BCIN_15g01180), BcSdhB (BCIN_01g04980), BcSdhC (BCIN_02g03080) and BcS$\mathrm{dhD}$ (BCIN_05g04430). The nucleotide sequences coding for these Sdh subunits were amplified in six FluR isolates and six FluS isolates using the corresponding specific primer pairs (Additional file 1: Table S1) and Phanta Max PCR kit (Vazyme, Nanjing, China). The PCR reactions were conducted using the following procedure: an initial denaturation at $95^{\circ} \mathrm{C}$ for $5 \mathrm{~min}$, followed by 35 cycles of denaturation at $95^{\circ} \mathrm{C}$ for $30 \mathrm{~s}$, annealing at $53^{\circ} \mathrm{C}$ for $30 \mathrm{~s}$, extension at $72{ }^{\circ} \mathrm{C}$ for $1-3 \mathrm{~min}$, and a final extension at $72{ }^{\circ} \mathrm{C}$ for $10 \mathrm{~min}$. The PCR products were then purified and sequenced. DNA sequences coding for the Sdh subunits were analyzed using Bioedit software (http://www. mbio.ncsu.edu/BioEdit/bioedit.html).

\section{Abbreviations \\ AEA: Alkylester agar medium; $\mathrm{EC}_{50}$ : Effective concentration for $50 \%$ inhibition of mycelial growth; FluR: Fluxapyroxad-resistant; FluS: Fluxapyroxad-sensitive; MM: Minimal medium; PCR: Polymerase chain reaction; PDA: Potato dextrose agar; PDB: Potato dextrose broth; PIMG: Percentage of inhibition of mycelial radial growth; Qols: Quinone outside inhibitors; RF: Resistance factor; Sdh: Suc- cinate dehydrogenase; SDHI: Succinate dehydrogenase inhibitor; WA: Water agar medium}

\section{Supplementary Information}

The online version contains supplementary material available at https://doi. org/10.1186/s42483-022-00107-3.

Additional file 1: Table S1. Primers used in this study.

\section{Acknowledgements}

Not applicable.

\section{Authors' contributions}

WS and ZM conceived and designed the experiments. KL, ZW and WS carried out the experiments. All authors analyzed the data. $\mathrm{KL}, \mathrm{ZM}$ and WS wrote the manuscript. All authors read and approved the final manuscript.

Funding

This research was supported by the National Natural Science Foundation of China (31901828)

Availability of data and materials Not applicable.

\section{Declarations}

Ethics approval and consent to participate Not applicable.

Consent for publication

Not applicable.

Competing interests

The authors declare that they have no competing interests.

Received: 18 October 2021 Accepted: 11 January 2022

Published online: 24 January 2022

\section{References}

Chen WC, Wei LL, Zhao WC, Wang BR, Zheng HH, Zhang PC, et al. Resistance risk assessment for a novel succinate dehydrogenase inhibitor pydiflumetofen in Fusarium asiaticum. Pest Manag Sci. 2021;77(1):538-47. https://doi.org/10.1002/ps.6053. 
Fan F, Hamada MS, Li N, Li GQ, Luo CX. Multiple fungicide resistance in Botrytis cinerea from greenhouse strawberries in Hubei Province, China. Plant Dis. 2017;101(4):601-6. https://doi.org/10.1094/PDIS-09-16-1227-RE.

Fernández-Ortuño D, Bryson PK, Grabke A, Schnabel G. First report of fludioxonil resistance in Botrytis cinerea from a strawberry field in Virginia. Plant Dis. 2013;97(6):848-9. https://doi.org/10.1094/PDIS-01-13-0012-PDN.

Hägerhäll C. Succinate: quinone oxidoreductases: variations on a conserved theme. Biochim Biophys Acta Bioenerg. 1997;1320(2):107-41. https://doi. org/10.1016/S0005-2728(97)00019-4.

Hahn M. The rising threat of fungicide resistance in plant pathogenic fungi: Botrytis as a case study. J Chem Biol. 2014;7:133-41. https://doi.org/10. 1007/s12154-014-0113-1.

Lalève A, Gamet S, Walker A, de Bieu D, Toquin V, Fillinger S. Site-directed mutagenesis of the $\mathrm{P} 225, \mathrm{~N} 230$ and $\mathrm{H} 272$ residues of succinate dehydrogenase subunit B from Botrytis cinerea highlights different roles in enzyme activity and inhibitor binding. Environ Microbiol. 2014;16(7):2253-66. https://doi.org/10.1111/1462-2920.12282.

Leroux P, Fritz R, Debieu D, Albertini C, Lanen C, Bach J, et al. Mechanisms of resistance to fungicides in field strains of Botrytis cinerea. Pest Manage Sci. 2002;58(9):876-88. https://doi.org/10.1002/ps.566.

Leroux P, Gredt M, Leroch M, Walker AS. Exploring mechanisms of resistance to respiratory inhibitors in field strains of Botrytis cinerea, the causal agent of gray mold. Appl Environ Microbiol. 2010;76(19):6615-30. https://doi.org/ 10.1128/AEM.00931-10

Li W, Wu Y, Yuan M, Liu X. Fluxapyroxad induces developmental delay in zebrafish (Danio rerio). Chemosphere. 2020;256: 127037. https://doi.org/ 10.1016/j.chemosphere.2020.127037.

Lin H, Lin F, Yuan J, Cui F, Chen J. Toxic effects and potential mechanisms of fluxapyroxad to zebrafish (Danio rerio) embryos. Sci Total Environ. 2021;2021: 144519. https://doi.org/10.1016/j.scitotenv.2020.144519.

Petrasch S, Knapp SJ, van Kan JAL, Blanco-Ulate B. Grey mould of strawberry, a devastating disease caused by the ubiquitous necrotrophic fungal pathogen Botrytis cinerea. Mol Plant Pathol. 2019;20(6):877-92. https://doi.org/ 10.1111/mpp.12794.

Shao W, Sun J, Zhang X, Chen C. Amino acid polymorphism in succinate dehydrogenase subunit $C$ involved in biological fitness of Botrytis cinerea. Mol Plant-Microbe Interact. 2020;33(4):580-9. https://doi.org/10.1094/ MPMI-07-19-0187-R.

Shao W, Zhao Y, Ma Z. Advances in understanding fungicide resistance in Botrytis cinerea in China. Phytopathology. 2021;111(3):455-63. https://doi. org/10.1094/PHYTO-07-20-0313-IA.

Sierotzki H, Scalliet G. A review of current knowledge of resistance aspects for the next-generation succinate dehydrogenase inhibitor fungicides. Phytopathology. 2013;103(9):880-7. https://doi.org/10.1094/ PHYTO-01-13-0009-RVW.

Veloukas T, Markoglou AN, Karaoglanidis GS. Differential effect of SdhB gene mutations on the sensitivity to SDHI fungicides in Botrytis cinerea. Plant Dis. 2013;97(1):118-22. https://doi.org/10.1094/PDIS-03-12-0322-RE.

Wang B, Lou T, Wei L, et al. Biochemical and molecular characterization of Alternaria alternata isolates highly resistant to procymidone from broccoli and cabbage. Phytopathol Res. 2021;3:15. https://doi.org/10.1186/ s42483-021-00092-z.

Williamson B, Tudzynski B, Tudzynski P, van Kan JAL. Botrytis cinerea: the cause of grey mould disease. Mol Plant Pathol. 2007;8(5):561-80. https://doi. org/10.1111/j.1364-3703.2007.00417.x.

Yang D, Zhao B, Fan Z, Yu B, Zhang N, Li Z, et al. Synthesis and biological activity of novel succinate dehydrogenase inhibitor derivatives as potent fungicide candidates. J Agric Food Chem. 2019;67(47):13185-94. https:// doi.org/10.1021/acs.jafc.9b05751.

Yin YN, Kim YK, Xiao CL. Molecular characterization of boscalid resistance in field isolates of Botrytis cinerea from apple in Washington State. Phytopathology. 2011;101(8):986-95. https://doi.org/10.1094/PHYTO-01-11-0016.

Yin WX, Adnan M, Shang Y, Lin Y, Luo CX. Sensitivity of Botrytis cinerea from nectarine/cherry in China to six fungicides and characterization of resistant isolates. Plant Dis. 2018;102(12):2578-85. https://doi.org/10.1094/ PDIS-02-18-0244-RE.

Zhang Y, Mao CX, Zhai XY, Jamieson PA, Zhang CQ. Mutation in cyp51b and overexpression of cyp51 $a$ and cyp $51 b$ confer multiple resistant to DMls fungicide prochloraz in Fusarium fujikuroi. Pest Manag Sci. 2021;77(2):824-33. https://doi.org/10.1002/ps.6085.
Zhou MG, Ye ZY, Liu JF. Progress of fungicide resistance research. J Nanjing Agric Univ. 1994;17(3):33-41. https://doi.org/10.7685/j.issn.1000-2030. 1994.03.005(inChinese).

\section{Publisher's Note}

Springer Nature remains neutral with regard to jurisdictional claims in published maps and institutional affiliations.
Ready to submit your research? Choose BMC and benefit from:

- fast, convenient online submission

- thorough peer review by experienced researchers in your field

- rapid publication on acceptance

- support for research data, including large and complex data types

- gold Open Access which fosters wider collaboration and increased citations

- maximum visibility for your research: over $100 \mathrm{M}$ website views per year

At BMC, research is always in progress.

Learn more biomedcentral.com/submissions 